\title{
UMA ABORDAGEM SOBRE A INTER-RELAÇÃO DE CITOCINAS NA ARTRITE
} REUMATÓIDE.

Diego Lucas de Campos (CAMPOS, D. L.) ${ }^{1}$

Leandro Fantin de Pontes (PONTES, L. F.) ${ }^{2}$

Fernanda Torres ${ }^{3}$

Jucélia Nunes da Silva ${ }^{4}$

André Tomaz Terra Júnior (TERRA JUNIOR, A. T.) ${ }^{5}$

\section{RESUMO}

O sistema imunológico é importante mecanismo de defesa e proteção do corpo contra as ações de agentes internos e externos, contudo, tal sistema pode atuar contra substâncias, células, órgão ou tecidos do próprio organismo, processo este denominado auto-imunidade, importante na regulação homeostática, que quando se desenvolve de forma patológica é capaz de gerar danos irreparáveis ao organismo, ocasionando as doenças auto-imunes. A artrite reumatóide é uma doença auto-imune, de etiologia exata ainda desconhecida, caracterizada pela ação inflamatória e degenerativa dos ossos e cartilagens das articulações, onde durante o desenvolvimento do processo inflamatório ocorre à síntese em grande escala de mediadores químicos denominados citocinas, principalmente as interleucinas IL-1, IL-6 e fator de necrose tumoral TNF-alfa. O objetivo deste trabalho foi descrever através de pesquisa sobre forma de revisão de literatura a relação entre artrite reumatóide e a ação das citocinas. A artrite reumatóide é uma doença que apresenta alta taxa de morbidade e é fator de risco para diversas doenças. A ação inflamatória da artrite reumatóide envolve uma série de processos, dentre os quais as ações de mediadores químicos como as citocinas desempenham papel fundamental durante o quadro inflamatório, consequentemente contribuindo para o quadro evolutivo crônico da doença.

Palavras-chave: artrite reumatóide, citocinas, auto-imunidade, inflamação.

\footnotetext{
${ }^{1}$ Discente do Curso de graduação em Farmácia da FAEMA.

${ }^{2}$ Discente do Curso de graduação em Farmácia da FAEMA.

${ }^{3}$ Especialista em Hematologia, Professora do Curso de Farmácia na Faculdade de Educação e Meio Ambiente - FAEMA, Ariquemes - RO;
} 
${ }^{4}$ Especialista em Análises Clínicas, Professora do Curso de Farmácia na Faculdade de Educação e Meio Ambiente FAEMA, Ariquemes - RO;

${ }^{5}$ Farmacêutico Industrial / Medicamentos, Mestre em Oncologia Clínica, Terapia Celular e Células Tronco pela Faculdade de Medicina de Ribeirão Preto - FMRP -USP, Docente do Curso de Graduação em Farmácia da FAEMA.

\title{
AN APPROACH ON CYTOKINE INTER- RELATIONSHIP IN RHEUMATOID ARTHRITIS.
}

\begin{abstract}
The immune system is important defense mechanism and body protection against the actions of internal and external agents, however, such a system can act against substances, cells, organs or tissues of the organism itself, a process called autoimmunity, important in the homeostatic regulation which develops when pathologically is capable of generating irreparable damage to the body, causing auto-immune diseases. Rheumatoid arthritis is an autoimmune disease, exact unknown etiology characterized by inflammatory and degenerative action of bones and cartilages of the joints, where during the development of the inflammatory process occurs for large-scale synthesis of chemical mediators called cytokines, especially interleukins IL-1, IL-6 and tumor necrosis factor TNF-alpha. The aim of this study was to describe through research on the form of literature review the relationship between rheumatoid arthritis and the action of cytokines. Rheumatoid arthritis is a disease with high morbidity and is a risk factor for many diseases. The inflammatory action of rheumatoid arthritis involves a series of processes, among which the actions of chemical mediators such as cytokines play a key role in the inflammatory process, thus contributing to chronic evolutionary picture of the disease.
\end{abstract}

Keywords: rheumatoid arthritis, cytokines, autoimmunity, inflammation.

\section{INTRODUÇÃO}

O sistema imune é um mecanismo importante e complexo do organismo humano, responsável pela proteção contra a ação de agentes estranhos ao corpo, que de alguma forma podem trazer distúrbios a homeostase, como: alimentos, substâncias químicas, pólen, poeira, drogas, dentre outros. No entanto, além dos agentes externos, o sistema imune é capaz de reconhecer agentes do 
próprio organismo como estranhos ou prejudiciais, processo este descrito como auto-imunidade, que pode trazer consequiências mínimas ou insignificantes ao ponto de vista patológico, mas também pode acarretar consequências catastróficas responsáveis pelo desenvolvimento de doenças denominadas doenças auto-imunes ${ }^{(1)}$.

A artrite reumatóide (AR) é uma doença auto-imune caracterizada pela ação inflamatória, geralmente crônica, que acomete as articulações, onde o próprio sistema imune do paciente é o responsável pela ação degenerativa das articulações ${ }^{(2)}$, afetando o tecido ósseo e cartilaginoso de forma irreversível ${ }^{(3)}$, ocasionando danos estruturais e anatômicos, podendo causar deficiência física, comprometendo a qualidade de vida do paciente ${ }^{(4)}$.

Além das complicações sistêmicas, a AR está associada como fator de risco para diversas donças cardiovasculares, como infarto agudo do miocáridio, aterosclerose coronária e extracoronária, dentre outras, fatos estes, relacionados aos processos inflamatórios sistêmicos que se desenvolvem ao longo da patogênia inflamatória ${ }^{(5)}$.

Quanto a prevelência, a AR afeta em média de 0.5 a $1.0 \%$ da população global, com maior incidência em pessoas do sexo feminino, com idade superior aos 40 anos, no entanto, as complicações iniciais da doenças começam a se desenvolver durante a juventude, com manifestações dolorosas ${ }^{(6)}$.

A etiologia ainda é desconhecida, porém, estudos demonstram que a AR está relacionada a um antígeno ainda não identificado que é responsável por desencadear o processo inflamatório sinovial, bem como, a relação com fatores genéticos, principalmente ao complexo de histocompatibilidade (MHC) ${ }^{(7)}$, e a presença de citocinas e moleculas pró-inflamatórias ${ }^{(2)}$.

Durante os processos inflamatórios ocorre uma grande produção de mediadores quimicos de origem proteíca, denominados citocinas, responsáveis dentre outras funções, pela atração leucocitária para o local da inflamação, potencializando o processo de defesa do organismo ${ }^{(8)}$.

Atualmente já foram identificadas mais de 100 variações de citocinas e outros fatores que estão diretamente relacionados ao desenvolvimento da AR, devendo-se destacar principalmente o fator de necrose tumoral TNF-alfa, citicina presente na maioria dos casos de degeneração articular ${ }^{(9)}$.

As citocinas são mediadores soluveis, de composição proteica, com baixo peso molecular, que são sintetizados por todas as células do sistema imune inato e adquirido, principalmente por células $\mathrm{T}^{(10)}$, responsáveis por regular e conduzir a resposta inflamatória, no entanto, a sua produção de forma desordenada pode ocasionar desequilibrio homeostático e desencadear distúrbios e complicações metabólicas ${ }^{(11)}$. 


\section{MATERIAIS E MÉTODOS}

O presente estudo foi realizado sobre a forma de revisão de literatura de carater exploratória, com intuito de descrever a relação entre artrite reumatóide e citocinas.

A pesquisa ocorreu através da leitura de artigos científicos, monografias e teses desponibilizados nos bancos de dados da biblioteca virtual SciELO (http://www.scielo.com.br), PubMed Central (http://www.pubmedcentral.nih.gov), Biblioteca Virtual de Saúde (http://www.bvsalud.org), bem como, artigos de outras bases de dados, que tivessem relação direta ou indireta ao assunto discutido. Foram selecionados artigos originais, revisão bibliográfica, estudo de caso, etc. Os idiomas selecionados foram português, inglês e espanhol, publicados no período de 2000 a 2016, utilizando-se os descritores: artrite reumatóide, citocinas, inflamação, mediadores inflamatórios.

Após a análise e leitura foram selecionados os artigos que apresentaram maior relevância ao estudo proposto e colocados em discussão.

\section{REVISÃO DE LITERATURA}

\subsection{PRINCIPAIS ASPECTOS DA ARTRITE REUMATÓIDE}

A artrite reumatóide AR é uma doença inflamatória crônica de origem auto-imune que acomete as articulações, geralmente das regiões periféricas ${ }^{(5)}$, com alta capacidade de degeneração irreversível do tecido ósseo, cartilaginoso e estruturas adjacentes ${ }^{(3)}$.

Durante a evolução do quadro reumático o paciente é acometido por disfunção articular progressiva, que com o passar do tempo as atividades cotidianas básicas ficam gradativamente prejudicadas ${ }^{(12)}$.

É uma doença pouco comum, com incidência global de 0,5 a $1 \%$, que afeta principalmente mulheres com idade superior a 40 anos, no entanto, a sintomatologia pode ser observada durante a juventude ${ }^{(6)}$.

No contexto nacional, há poucos estudos que determinam a prevalência da AR, no entanto, a limitada literatura cogita-se uma estimativa de aproximadamente 0,2 a $1 \%$ da população brasileira sofra da doença ${ }^{(13)}$. As características laboratoriais em pacientes brasileiros são pouco esclarecidas, 
no entanto, os poucos registros demonstram manifestações laboratoriais entre a população brasileira são semelhantes as demais populações ${ }^{(14)}$.

Os principais sintomas articulares da AR envolvem dor e edema, proveniente de processo inflamatório, podendo haver rigidez matinal, nódulos reumatóides além de outras manifestações sugestivas de acometimento de estruturas articulares, bem como, a associação a outras manifestações como fadiga, fibromialgia, anemia, vasculite cutânea, dentre outras ${ }^{(3)}$.

A AR é fator de risco para doenças cardiovasculares como infarto agudo do miocárdio, ateroscleroma acelerada coronária e extracoronária, em virtude dos processos inflamatórios sistêmicos que são desencadeados ao período que a doença se desenvolve, também $\mathrm{s}$ fator de risco para síndromes metabólicas como obesidade, dislipidemia, hipertensão e hiperglicemia ${ }^{(5)}$.

\subsection{PROPRIEDADES GERAIS DAS CITOCINAS}

As citocinas são proteínas de baixo peso molecular, solúveis, produzidas geralmente após ou durante uma resposta do organismo a antígenos, que atuam na regulação química das respostas imunológicas ${ }^{(13)}$. Possuem diversas atribuições biológicas e são produzidas por diversas células, principalmente por linfócitos, monócitos e macrófagos ${ }^{(14)}$, e desempenham papel fundamental na condução das células imunológicas, principalmente os leucócitos, até os locais onde está ocorrendo à resposta imune ${ }^{(15)}$.

Há um tipo específico de citocinas denominadas interleucinas e interferons, que também são secretados por células imunológicas e desempenham importante papel nas respostas imunes e inflamatórias ${ }^{(16)}$.

Atuam com eficiência em pequenas concentrações, são raramente encontradas em indivíduos saudáveis, em contrapartida, estão presentes em altas concentrações em indivíduos que apresentam quadros infecciosos, inflamatórios e doenças crônicas ${ }^{(14)}$.

Esses peptídeos, denominados citocinas, constituem um grupo de fatores extracelulares produzidos pelos leucócitos, que são definidas e classificadas em diversas categorias de acordo com suas funções, são elas: interferons (IFN), interleucinas (IL), fator estimulador de colonias (CSF), fator de necrose tumoral (TNF), fator de transformação de crescimento (TGF), dentre outros ${ }^{(17)}$.

\subsection{RELAÇÃO ENTRE CITOCINAS E ARTRITE REUMATÓIDE}


Em regra, a resposta inflamatória é iniciada por uma fase aguda, onde o paciente apresenta febre, produção de diversos hormônios, leucocitose e elevada produção de proteínas específicas pelos hepatócitos, bem como a produção de importantes substâncias que subsidiaram a resposta inflamatória como citocinas, interleucinas IL-1 e IL-6, além do fator de necrose tumoral TNF-alfa (18)

Nos processos inflamatórios reumatóides, estudos demonstram a existências de um antígeno, ainda desconhecido, responsável por desencadear os processos inflamatórios nas articulações ${ }^{(7)}$, e que este antígeno ativa linfócitos TCD4, por intermédio de células apresentadoras de antígeno, aumentando a síntese de linfócitos T, que consequentemente aumentam a ploriferação e diferenciação de linfócitos B, ocorrendo constante ativação de macrófagos, que além de seu papel fundamental, ou seja, fagocitose, esses macrófagos também são responsáveis pela liberação de citocinas, resultando em um processo inflamatório crônico com a presença de diversas outras células inflamatórias, que também irão liberar quimiocinas, citocinas, prostaglandinas, leucotrienos, dentre outras substâncias pró e antiinflamatórias, deixando o processo inflamatório cada vez mais complexo e lesivo aos tecidos locais ${ }^{(2)}$.

Fatores genéticos ligados ao complexo de histocompatibilidade (MHC), também podem estar associados a AR, pois o MHC é responsável pela apresentação de antígenos para os linfócitos TCD4+, levando a uma resposta auto-imune imediata por linfócitos T, resultando em um processo inflamatório lesivo aos ossos e cartilagens sinoviais ${ }^{(7)}$. O MHC esta frequentemente presente nos casos de doenças auto-imunes, pois estabelece uma importante função na apresentação de antígeno, estabelecendo ligação entre resposta imunológica inata e adaptativa ${ }^{(19)}$.

O fator de necrose tumoral TNF-alfa está presente nos processos inflamatórios articulares e pode ser associado aos casos de destruição tecidual, principalmente por sua ação na ativação de osteoclastos ${ }^{(9)}$, células originadas dos monócitos e macrófagos, responsáveis pela reabsorção óssea ${ }^{(20)}$. Estudos in vitro e processos experimentais in vivo demonstraram que o TNF-alfa apresenta capacidade lesiva sobre o tecido cartilaginoso ${ }^{(2)}$. O TNF-alfa quando presente, também é responsável pela estimulação de enzimas capazes de ampliar a resposta inflamatória já instalada ${ }^{(20)}$, sendo capaz também de induzir outras citocinas, como as interleucinas IL-1 e IL-6, bem como, estimular a permeabilidade vascular e atrair leucócitos ${ }^{(2)}$.

\subsubsection{PRINCIPAIS CITOCINAS ENVOLVIDAS AR}


As citocinas IL-1, IL-6 e TNF-alfa são importantes mecanismos que facilitam o processo de resposta inflamatória da imunidade inata, pois, são responsáveis por uma extensa série de processos e eventos biológicos que são desencadeados ao longo da resposta imune. Em sua maioria são especialmente produzidas por células fagocitárias, e desempenham papel fundamental no processo febril, na fase inflamatória aguda e na síntese de proteínas da fase aguda dos processos inflamatórios, e quimiotaxia de diversos subgrupos de leucócitos ${ }^{(1)}$.

Atuam basicamente na influência da síntese e dinâmica de leucócitos a níveis séricos, contribuindo para a migração de células para os mais diversos tecidos do corpo ${ }^{(23)}$.

\subsubsection{IL-1}

As interleucinas IL-1 são formadas principalmente por monócitos e macrófagos, no entanto, também podem ser obtidas de outros tipos celulares como células endoteliais, fibroblastos e alguns linfócitos ${ }^{(21)}$.

São encontradas facilmente no líquido sinovial e é importante mediador inflamatório, pois têm alta capacidade de ativar monócitos, macrófagos e linfócitos, além de estimular a síntese de diversas moléculas inflamatórias, citocinas, quimiocinas, prostaglandinas e óxido nítrico ${ }^{(2)}$.

Também induzem os linfócitos T CD4+, que consequentemente induzirá e estimulará a proliferação e estimulação dos linfócitos $\mathrm{B}$, neutrófilos, monócitos/macrófagos, aumentando a atividade fogocitária e quimiotatica, estimula a adesão leucocitária, ativa os processos de coagulação e estimula as células do fígado a sintetizar proteínas importantes para a fase aguda dos processos inflamatórios ${ }^{(21)}$.

\subsubsection{IL-6}

Esta citocina é sintetizada principalmente por monócitos e linfócitos B e T, e é responsável pela resposta imunológica antígeno específico, atua como importante mediador da fase aguda dos processos inflamatórios, estimulando a síntese de proteínas pró-inflamatórias pelos hepatócitos, possui grande potencial de atração de eosinófilos, estimulam a produção das interleucinas IL-1 e TNF-alfa ${ }^{(21)}$, além de estimular e promover a proliferação dos linfócitos T e B ${ }^{(2)}$.

\subsubsection{TNF-alfa}


O fator de necrose tumoral TNF-alfa é sentetizado principalmente pelos macrófagos, porém, outras células imunológicas como linfócitos T, monócitos e células Natural Killer (NK) também podem sintetizar a substância após estímulo antigênico externo ${ }^{(21)}$. É um importante fator na estimulação da cicatrização de feridas, bem como na resposta imune, no entanto, quando em altas concentrações podem desencadear diversas complicações ${ }^{(25)}$.

O TNF-alfa é um importante indutor da síntese de citocinas inflamatórias do tipo IL-1 e IL6, potencializando a migração de leucócitos e ativando neutrófilos ${ }^{(2)}$. Apresenta como principal função a atividade citolítica de diversos tipos celulares ${ }^{(21)}$.

\section{CONCLUSÃO}

O sistema imunológico é um mecanismo importante que dispõe o organismo para o combate de doenças e tudo aquilo que é estranho ou anormal ao sistema fisiológico, no entanto, quando esse mecanismo de defesa age contra o próprio organismo acarreta uma série de complicações, muitas vezes irreversíveis, como as doenças auto-imunes

A artrite reumatóide é uma doença inflamatória crônica degenerativa que afeta pessoas a partir da quarta década de vida, se manifestando geralmente de forma dolorosa, comprometendo a atividade funcional das articulações acometidas, causando prejuízos à qualidade de vida do paciente.

Também é um fator de risco para outras doenças, devido aos processos inflamatórios decorrentes da patologia, e pela alta taxa de morbidade, que além de contribuir para problemas físicos pode ser atrelado a distúrbios psicológicos.

Quanto à relação entre a artrite reumatóide e as citocinas, observa-se que pelo fato de ser uma doença desencadeada por um processo inflamatório inicial, ainda não totalmente desvendado, as citocinas desempenham papel fundamental na evolução do quadro inflamatório, consequentemente no quadro evolutivo da doença, uma vez que, a presença dessas substâncias nas regiões sinoviais é primordial na indução da síntese e atração leucocitária, síntese de novas citocinas e outras substâncias pró-inflamatórias, que de certa forma agravam o processo inflamatório inicial, tornando-se assim uma espécie de "ciclo-inflamatório" que se agrava ao percurso do processo evolutivo da reação inflamatória inicial.

Vale ressaltar que existem poucos estudos sobre as manifestações laboratoriais da AR na população brasileira. Embora a escassa literatura demonstre semelhanças nas alterações 
biomoleculares entre brasileiros e demais população, se faz necessário um amplo estudo para um mapeamento detalhado dessas manifestações, principalmente quanto à presença das citocinas e demais mediadores inflamatórios que contribuem para o desenvolvimento da patologia, possibilitando novas descobertas e formas de tratamento, garantindo assim maior qualidade e expectativa de vida aos pacientes.

Portanto, é necessário a adoção de maiores investimentos e incentivo a estudos e pesquisas, no intuito de elucidar os fatos ainda pouco esclarecidos sobre a doença, os quais podem revelar mecanismos de tratamentos mais específicos e eficazes, uma vez que a AR é uma doença de evolução progressiva, devastadora, que acarreta sérios prejuízos a qualidade de vida dos pacientes.

\section{REFERÊNCIAS BIBLIOGRÁFICAS}

1. Benjamini E, Coico R, Sunshine G. Imunologia. 4th ed. Rio de Janeiro: Guanabara Koogan; 2002.

2. Da Silva MLF. Artrite Reumatóide: O papel das moléculas pró-inflamatórias e antiinflamatórias. 2010. Academia de Ciências e Tecnologia de São Jose do Rio Preto - Curso de Imunologia Clinica e Laboratórial.

3. Da Mota LMH, Laurindo IMM, Dos Santos Neto LL. Características demográficas e clínicas de uma coorte de pacientes com artrite reumatoide inicial. Rev Bras Reumatol. 2010; 50(3).

4. Corbacho MI, Dapueto JJ. Avaliação da capacidade funcional e da qualidade de vida de pacientes com artrite reumatoide. Rev Bras Reumatol. 2010; 50(1).

5. Da Cunha VR, Brenol CV, Brenol JCT, Xavier RM. Artrite reumatoide e síndrome metabólica. Rev Bras Reumatol. 2011; 51(3).

6. Dario AB, Külkamp W, Faraco HC, Gevaerd M, Domenech SC. Alterações psicológicas e exercício físico em pacientes com artrite reumatoide. Revista Motricidade. 2007 Set; 6(3).

7. Faleiro LR, Araújo LHR, Varavallo MA. A terapia anti-TNF-a na artrite reumatóide. Semina: Ciências Biológicas e da Saúde. 2011 Jun; 32(1).

8. Balbino CA, Pereira LM, Curi R. Mecanismos envolvidos na cicatrização: uma revisão. Revista Brasileira de Ciências Farmacêuticas. 2005 Mar.; 41(1).

9. Wiens A, Grochocki MC, Pontarolli DRS, Venson R, Correr CJ, Pontarolo R. Perfi 1 dos usuários de anticitocinas disponibilizadas pelo Sistema Único de Saúde no estado do Paraná para o tratamento da artrite reumatoide. Rev Bras Reumatol. 2012; 52(2).

10. Benjamini E, Coico R, Sunshine G. As citocinas. In Benjamini E, Coico R, Sunshine G. Imunologia. Rio de Janeiro - RJ: Guanabara Koogan; 2002. p. 288.

11. De Oliveira CMB, Sakata RK, Issy AMI, Gerola LR, Salomão R. Citocinas e Dor. Rev Bras Anestesiol. 2011; 61(2).

12. Deus Junior RS, Ferraz AL, Oesterreich SA, Schmitz WO, Shinzato MM. Caracterizac são de pacientes com artrite reumatoide quanto a fatores de risco para doenc, as vasculares cardíacas no Mato Grosso do Sul. Revista Brasileira de Reumatologia. 2015; 55(6). 
13. Dabés CGeS, Almeida AM, Acurcio FDA. Não adesão à terapia biológica em pacientes com doenças reumáticas no Sistema Único de Saúde em Minas Gerais, Brasil. Cad. Saúde Pública. 2015 Dez.; 31(12).

14. Da Mota LaH, Dos Santos Neto LL, Burlingame R, Ménard H, Laurindo IMM. Características laboratoriais de um grupo de pacientes com artrite reumatoide inicial. Revista Brasileira de Reumatologia. 2010 Ago; 50(4).

15. Pereira AB, De Rezende NA, Teixeira Junior AL, Teixeira MM, Silva ACS. Citocinas e quimiocinas no transplante renal. J. bras. nefrol. 2009 Dez.; 31(4).

16. Sarrouh BF, Rivaldi JD, Gambarato BC, Dos Santos DT, Da Silva SS. Importância das citoquinas no sistema imunológico - Parte I: propriedades e características funcionais. Revista Analytica. 2008 Jan.; 32.

17. Gonçalves RM, Teixeira AL, Campos WR, Oréfice F. O papel das quimiocinas nas uveítes. Arq. Bras. Oftalmol. 2007; 70(2).

18. Naoum PC. Citocinas e Interleucinas. 2009. Academia de Ciência e Tecnologia de São José do Rio Preto - SP.

19. Sarrouh BF, Rivaldi JD, Gambarato BC, Dos Santos DT, Da Silva SS. Importância das citoquinas no sistema imunológico Parte II: extração, purificação e análise. Revista Analytica. 2008 Mar.; 33.

20. Bilate AM. Inflamação, citocinas, proteínas de fase aguda e implicações terapêuticas. Temas de reumatologia clínica. 2007 Jun.; 8(2).

21. Cruvinel WdM, Mesquita Júnior D, Araújo JAP, Catelan TTT, De Souza AWS, Da Silva NP, et al. Sistema Imunitário - Parte I Fundamentos da imunidade inata com ênfase nos mecanismos moleculares e celulares da resposta inflamatória. Revista Brasileira de Reumatologia. 2010; 50(4).

22. Vitale RF, Ribeiro FAQ. O papel do Fator de Necrose Tumoral Alfa (TNF- $\alpha$ ) no processo de erosão óssea presente no colesteatoma adquirido da orelha média. Revista Brasileira DE Otorrinolaringologia. 2007 Jan./Fev.; 73(1).

23. Cruvinel WdM, Mesquita Júnior D, Araújo JAP, Catelan TTT, De Souza AWS, Da Silva NP, et al. Immune system: Part I. Fundamentals of innate immunity with emphasis on molecular and cellular mechanisms of inflammatory response. Revista brasileira de reumatologia. 2010 Ago; 50(4).

24. Varella PP, Forte WCN. Citocinas: revisão. Rev. bras. alergia imunopatol. 2001 Ago.; 24.

25. Azevedo VF, Silva MBG, Marinello DK, Dos Santos FD, Silva GBG. Leucopenia e trombocitopenia induzidas por etanercepte: relato de dois casos e revisão da literatura. Revista Brasileira de Reumatologia. 2012 Fev; 50(1). 